\title{
HLA-DR in Brazilian patients with polyarteritis nodosa (PAN) and microscopic polyangiitis (MPA)
}

\author{
Alzírton de Lira Freire ${ }^{\mathrm{a}, *}$, Roseneide Aparecida Conde ${ }^{\mathrm{a}}$, Manoel Barros Bertolo ${ }^{\mathrm{a}}$, \\ Lílian Teresa Lavras Costallat ${ }^{\mathrm{a}}$, Maurício Levy-Neto ${ }^{\mathrm{b}}$ and Sandra Regina Muchinechi Fernandes ${ }^{\mathrm{a}}$ \\ ${ }^{a}$ Department of Rheumatology, Faculty of Medical Sciences, State University of Campinas (UNICAMP), São \\ Paulo, Brazil \\ ${ }^{\mathrm{b}}$ State University of São Paulo, São Paulo, Brazil
}

\begin{abstract}
The aim of this study was to evaluate the frequency and clinical associations of HLA-DR alleles in Brazilian Caucasian patients with polyarteritis nodosa (PAN) or microscopic polyangiitis (MPA). We evaluated 29 Caucasian patients with vasculitis classified as PAN or MPA according to the American College of Rheumatology (ACR) 1990 Criteria, Chapel Hill Consensus Conference (CHCC) nomenclature for vasculitis and EULAR recommendations for conducting clinical studies in systemic vasculitis. HLA-DR alleles were typed using polymerase chain reaction-amplified DNA, hybridized with sequence-specific low resolution primers. DNA obtained from 59 Caucasian healthy blood donors were used as control. In order to evaluate if a specific HLA may have influence on the clinical profile of those diseases, we also divided the patients according to Birmingham vasculitis score (BVAS) and Five-Factors Score (FFS) at the time of diagnosis. Increased frequency of HLA-DRB1 $* 16(p=0.023)$ and DRB4*01 $(p=0.048)$ was found in patients with higher disease activity at the time of diagnosis (BVAS $\geqslant 22)$. Patients with less severe disease $(\mathrm{FFS}=0)$ had a higher frequency of HLA-DRB ${ }^{*} 03(p=0.011)$. Patients with gastrointestinal tract involvement had significantly increased frequency of HLA-DRB $1 * 11$ or B1*12 $(p=0.046), \mathrm{B} 1 * 13(p=0.021)$ and $\mathrm{B} 3(p=0.008)$. In contrast, patients with renal disease, had higher frequency of DRB $1 * 15$ or DRB $1 * 16(p=0.035)$ and B5 $(p=0.035)$. In the subgroup of patients with MPA, increased frequency of HLA-DRB1*15 was found in patients with BVAS $\geqslant 22(p=0.038)$ and FFS $\geqslant 1(p=0.039)$ suggesting that this allele is associated with more aggressive disease. Antineutrophil cytoplasmic antibodies (ANCA) negative MPA patients had significantly increased frequency of HLA-DRB $1 * 11$ or DRB $1 * 12$ when compared to ANCA positive patients $(p=0.023)$. Our results suggest that HLA-DR alleles may influence PAN and MPA clinical expression and outcome and that in MPA they participate in the mechanisms involved in the development to ANCA.
\end{abstract}

Keywords: Polyarteritis nodosa, microscopic polyangiitis, HLA, vasculitis, autoimmunity

\section{Introduction}

The auto-immune diseases are often associated with human leukocyte antigen (HLA) genes, which are encoded in the major histocompatibility complex (MHC). Their products, the HLA molecules, do participate in

* Corresponding author: Alzírton de Lira Freire, Departamento de Clínica Médica, Reumatologia, FCM/UNICAMP, Rua Alexander Fleming, 40 Campinas, São Paulo, Brazil. CEP: 13083-970, Caixa Postal: 6111. E-mail: apfreire@hotlink.com.br. antigen presentation and in the cell response, thus, playing an important role in the immune response. Polyarteritis nodosa (PAN) and microscopic polyangiitis (MPA) are uncommon systemic necrotizing vasculitis. Their underlying pathogenic mechanisms appear to be different, involving antineutrophilic cytoplasmic antibody (ANCA) in MPA and immune complexes in PAN [1-6]. The occurrence of these diseases in members of the same family and differences in their prevalence among population groups may suggest that environmental and genetic factors may play a role in their 
etiopathogenesis [4-14]. Up to now, it is unknown if polymorphisms related to HLA genes may cause susceptibility to the development of those diseases. HLADR alleles are the class II HLA genes most frequently associated to immunopathologic conditions such as rheumatoid arthritis. When we focus on systemic vasculitis, the association of HLA-DR alleles with PAN and MPA has been reported, but with variable results according with the studied population [4-6]. The aim of our study was to evaluate whether there is an association between HLA-DR alleles and PAN or MPA in Brazilian Caucasian patients and also whether this association differs according to clinical severity of those diseases.

\section{Methods}

\subsection{Patients and controls}

The study was carried out in 29 Caucasian patients with vasculitis classified as PAN not related to hepatitis B virus infection or MPA who were attended to at the university hospitals of the State University of Campinas and the State University of São Paulo. The classification was based on the American College of Rheumatology (ACR) 1990 criteria, Chapel Hill Consensus Conference (CHCC) nomenclature for vasculitis and EULAR recommendations for conducting clinical studies in systemic vasculitis [1-3]. The clinical and laboratorial features were evaluated retrospectively at the time of diagnosis. Disease activity at the time of diagnosis was evaluated by the Birmingham vasculitis activity score (BVAS) [15]. The Five-Factors Score (FFS) at the time of diagnosis was applied to evaluate the prognosis as previously reported [16]. According to the BVAS, the patients were divided into two groups: those with BVAS $<22$ and the other with BVAS $\geqslant$ 22 [17]. According to FFS, two classes of scores were defined: 0 , when no poor-prognosis factor was noted and $\geqslant 1$ when 1 or more factors were present [17] (Table 1). Blood samples of 59 unrelated Caucasian healthy blood donors from the Hemotherapy Center of the State University of Campinas were used as control. This study was reviewed and approved by the ethics committees of the institutions. Samples were obtained from the patients and controls after they had given written informed consent.

\subsection{HLA-DR typing}

HLA-DR alleles were typed using polymerase chain reaction-amplified DNA hybridized with sequencespecific low resolution DR primers (Dynal, Biotech Ltd. UK).

\subsection{Statistical analysis}

Statistical significance was evaluated by the $\chi^{2}$ test or Fisher's exact test. P values below 0.05 were considered statistically significant. Odds ratio (OR) was estimated using the SAS System computer program for Windows 8.2 (SAS Institute Inc., Cary, NC, USA).

\section{Results}

No statistically significant association was found in the frequency of HLA-DR alleles in patients compared to healthy controls. Table 2 shows the associations among specific HLA-DR antigens, activity index score, prognosis factors and clinical manifestations in the whole group of the patients (PAN and MPA). Increased frequency of HLA-DRB $1 * 16(p=0.023)$ and DRB4*01 ( $p=0.048)$ was found in patients with a higher score of disease activity at the time of diagnosis $($ BVAS $\geqslant 22$ ). Patients with no severity score (FFS $=0)$ had increased frequency of HLA-DRB $1 * 03$. The frequency of HLA-DRB $1 * 11$ or B $1 * 12(p=0.046)$, $\mathrm{B} 1 * 13(p=0.021)$ and B3 $(p=0.008)$ was significantly increased in patients with gastrointestinal (GI) tract involvement and DRB1*15 or DRB1*16 ( $p=$ $0.035)$ and B5 $(p=0.035)$ in those with renal disease. Regarding PAN patients, no statistically significant association was found. MPA patients with BVAS $\geqslant 22$ $(p=0.038)$ and FFS $\geqslant 1(p=0.039)$ had statistically significant increased frequency of HLA-DRB1*15. We found an increased statistically significant frequency of HLA-DRB $1 * 15$ or DRB $1 * 16(p=0.033)$ and B5 $(p=0.033)$ in those patients with renal involvement and also HLA-DRB $1 * 11$ or DRB $1 * 12(p=0.023)$ in those with negative ANCA (Table 3 ).

\section{Discussion}

There are few reports about PAN affecting relatives, including identical twins [7-11]. Rottem et al. described three daughters of a healthy couple with PAN and the girls share the same HLA haplotype (DR7, 
Table 1

Clinical features, BVAS and FFS $(\%)$ at the time of diagnosis

\begin{tabular}{lccc}
\hline Characteristic & Total group $(n=29)$ & PAN $(n=19)$ & MPA $(n=10)$ \\
\hline Mean Age (range) (years) & $38(12-70)$ & $37(12-52)$ & $39(24-70)$ \\
Gender M/F & $11 / 18$ & $8 / 11$ & $3 / 7$ \\
General symptoms & $19(65)$ & $14(74)$ & $5(50)$ \\
Arthralgias/arthritis & $19(65)$ & $10(53)$ & $9(90)$ \\
Myalgia & $19(65)$ & $15(79)$ & $4(40)$ \\
CNS involvement & $6(21)$ & $6(31)$ & $0(00)$ \\
Peripheral neuropathy & $16(55)$ & $10(53)$ & $6(60)$ \\
Skin lesions & $22(76)$ & $14(74)$ & $8(80)$ \\
Lung involvement & $4(14)$ & $1(5)$ & $3(30)$ \\
GI tract involvement & $12(41)$ & $6(31)$ & $6(60)$ \\
Cardiovascular & $3(10)$ & $1(5)$ & $2(20)$ \\
Renal & $14(48)$ & $10(53)$ & $4(40)$ \\
Eye involvement & $5(17)$ & $2(10)$ & $3(30)$ \\
BVAS $<22$ & $17(59)$ & $10(53)$ & $7(70)$ \\
BVAS $\geqslant 22$ & $12(41)$ & $9(47)$ & $3(30)$ \\
FFS $=0$ & $14(48)$ & $10(53)$ & $4(40)$ \\
FFS $\geqslant 1$ & $15(52)$ & $9(47)$ & $6(60)$ \\
\hline
\end{tabular}

CNS, Central Nervous System; GI, gastrointestinal; BVAS, Birmingham Vasculitis Activity Score; FFS, Five-Factors Score; PAN, polyarteritis nodosa; MPA, microscopic polyangiitis.

Table 2

Alleles HLA-DR in total group

\begin{tabular}{|c|c|c|c|c|}
\hline Allele frequency & $\begin{array}{c}\mathrm{BVAS} \geqslant 22(\%) \\
(n=12)\end{array}$ & $\begin{array}{c}\mathrm{BVAS}<22(\%) \\
(n=17)\end{array}$ & $\begin{array}{c}\text { Controls }(\%) \\
(n=59)\end{array}$ & $\mathrm{p}$ \\
\hline $\mathrm{DRB} 1 * 16$ & $3(25)$ & $0(0)$ & $2(3)$ & $0.023^{*}$ \\
\hline \multirow[t]{2}{*}{ DRB4*01 } & $7(58)$ & $3(18)$ & $28(47)$ & $0.048^{*}$ \\
\hline & $\begin{array}{c}\mathrm{FFS} \geqslant 1(\%) \\
(n=15)\end{array}$ & $\begin{array}{c}\mathrm{FFS}=0(\%) \\
(n=14)\end{array}$ & $\begin{array}{l}\text { Controls }(\%) \\
(n=59)\end{array}$ & $\mathrm{p}$ \\
\hline \multirow[t]{2}{*}{$\mathrm{DRB} 1 * 03$} & $0(0)$ & $6(43)$ & $11(19)$ & $0.011^{*}$ \\
\hline & $\begin{array}{c}\mathrm{GIT}+(\%) \\
(n=12)\end{array}$ & $\begin{array}{l}\mathrm{GIT}-(\%) \\
(n=17)\end{array}$ & & $\mathrm{p}$ \\
\hline $\mathrm{DRB} 1 * 11 / \mathrm{DRB} 1 * 12$ & $7(58)$ & $3(18)$ & & $0.046^{*}$ \\
\hline $\mathrm{DRB} 1 * 13$ & $4(33)$ & $0(0)$ & & $0.021^{*}$ \\
\hline \multirow[t]{2}{*}{ DRB3 } & $11(92)$ & $7(41)$ & & $0.008^{*}$ \\
\hline & $\begin{array}{l}\text { Renal }+(\%) \\
\quad(n=14)\end{array}$ & $\begin{array}{l}\text { Renal }-(\%) \\
\quad(n=15)\end{array}$ & & $\mathrm{p}$ \\
\hline DRB1*15/DRB1*16 & $6(43)$ & $1(7)$ & & $0.035^{*}$ \\
\hline DRB5 & $6(43)$ & $1(7)$ & & $0.035^{*}$ \\
\hline
\end{tabular}

${ }^{*} p<0.05$.

BVAS, Birmingham vasculitis activity score; FFS, Five-Factors Score; GIT, gastrointestinal tract; +, with involvement; -, without involvement.

DR53) of their healthy father [10]. Mason et al. reported two Asian siblings affected by PAN and they had the same HLA haplotype (DR5) as their healthy mother [11]. MPA has also been reported in members of the same family [4,12-14]. HLA studies involving groups of unrelated individuals with PAN or MPA are few. Boki et al., Spencer et al. and Papasteriades et al. found a decreased frequency of HLA-DRB1*03 in patients with PAN and MPA [18, 20,21]. Other reports failed to find association between HLA antigens and PAN [9,19]. Previous studies in Caucasian patients with ANCA-associated sys- temic vasculitis (AASV) showed a negative association with HLA-DR6-DRB1*13, suggesting a protective effect against the development of those diseases [22,23]. On the other hand, an increased frequency of HLADRB1*0901 has been reported in Asian patients with MPA [24]. Some of these studies were published before the Chapel Hill nomenclature was established and probably MPA patients were evaluated together with patients with PAN. In our study, we evaluated PAN and MPA patients together as a whole group and also each one of the disease just to compare with those previous reports. We did not find association between HLA-DR 
Table 3

Alleles HLA-DR in MPA

\begin{tabular}{|c|c|c|c|c|}
\hline Allele frequency & $\begin{array}{c}\text { BVAS } \geqslant 22(\%) \\
(n=3)\end{array}$ & $\begin{array}{c}\text { BVAS }<22(\%) \\
(n=7)\end{array}$ & $\begin{array}{l}\text { Controls }(\%) \\
(n=59)\end{array}$ & $\mathrm{p}$ \\
\hline DRB1*15 & $2(67)$ & $1(14)$ & $6(10)$ & $0.038^{*}$ \\
\hline DRB1*15 & $\begin{array}{c}\text { FFS } \geqslant 1(\%) \\
(n=6) \\
3(50)\end{array}$ & $\begin{array}{c}\text { FFS }=0(\%) \\
(n=4) \\
0(0)\end{array}$ & $\begin{array}{c}\text { Controls }(\%) \\
(n=59) \\
6(10)\end{array}$ & $0.039^{*}$ \\
\hline & $\begin{array}{c}\text { Renal }+(\%) \\
(n=4)\end{array}$ & $\begin{array}{l}\text { Renal }-(\%) \\
(n=6)\end{array}$ & & $\mathrm{p}$ \\
\hline DRB $1 * 15 / \mathrm{DRB} 1 * 16$ & $3(75)$ & $0(0)$ & & $0.033^{*}$ \\
\hline DRB5 & $3(75)$ & $0(0)$ & & $0.033^{*}$ \\
\hline $\mathrm{DRB} 1 * 11 / \mathrm{DRB} 1 * 12$ & $\begin{array}{c}\mathrm{ANCA}+(\%) \\
(n=4) \\
1(25)\end{array}$ & $\begin{array}{c}\text { ANCA }-(\%) \\
(n=4) \\
4(100)\end{array}$ & & $0.023^{*}$ \\
\hline
\end{tabular}

${ }^{*} p<0.05$.

BVAS, Birmingham vasculitis activity score; FFS, Five-Factors Score; Renal +, with renal involvement; Renal -, without renal involvement; ANCA +, positive ANCA; ANCA -, negative ANCA.

alleles and the diagnosis of PAN or MPA.

There are no studies about association among a specific HLA-DR allele, prognosis factors and activity index score in patients with PAN and MPA. Papasteriades et al. described 5 out of 6 patients with MPA and rapidly progressive renal disease who were positive for HLA-DR5 and they suggested that this specific HLA may be related to a more severe disease [21]. In our total group of patients, an increased frequency of DRB $1 * 11$ or DRB1*12, previously typed as HLADR5, was found related to GI tract disease, a wellknown severe manifestation of the diseases. We also found an increased frequency of HLA-DRB $1 * 16$ and $\mathrm{B} 4 * 01$ in those patients with more disseminated disease measured by BVAS. In contrast, HLA-DRB $1 * 03$ was more frequent in those with mild disease. An increased frequency of HLA-DRB $1 * 11$ or B $1 * 12, \mathrm{~B} 1 * 13$ and B3 was found in patients with GI tract involvement. HLA DRB $1 * 15$ or B1*16 and B5 was found in a higher frequency in patients with renal disease, especially in MPA patients. The renal and GI tract involvements contribute to a poor prognosis in PAN and MPA. Our results suggest that HLA-DR alleles may influence the clinical expression as well the outcome of PAN and MPA in Brazilian Caucasian patients.

Although histopathology of involved tissues in PAN and MPA are very similar, MPA is considered an ANCA associated systemic vasculitis (AASV) while PAN is mediated by an immune-complex. The group of AASV diseases includes in addition to MPA, two other types of vasculitis: Churg Strauss Syndrome and Wegener Granulomatosis. MPA differs from the other two in terms of a non-granulomatous histopathology and a lower frequency of ANCA. The small number of pa- tients with MPA of our series may limit the power of our results. However, we found an increased frequency of HLA-DRB $1 * 15$ in patients with BVAS $\geqslant 22$ and FFS $\geqslant 1$ suggesting that this allele may be associated with a spectrum of more aggressive disease. This finding may suggest that selected antigenic determinants may be involved in the development of the severe disease and also highlights the pathogenic role of T cells. We also found the interesting association of HLADRB $1 * 11$ or B1*12 with ANCA negative MPA. Up to now, HLA typing studies have not disclosed a definite association between ANCA and HLA in MPA. The ANCA antigen involved in MPA is mieloperoxidase (MPO). The interaction of MPO with these alleles in neutrophils membranes and endothelial cells is a topic that deserves further studies aiming for a better understanding of the mechanisms involved in the development of MPA.

In conclusion, our results suggest that HLA-DR alleles may influence PAN and MPA clinical expression and outcome and that in MPA they participate in the mechanisms involved in the development to ANCA.

\section{Acknowledgements}

The authors would like to thank Cleide Moreira Silva and Helymar da Costa Machado for the statistical analysis and FAEPEX for the financial support.

\section{References}

[1] R.W. Lightfoot Jr, B.A. Michel, D.A. Bloch et al., The American College of Rheumatology 1990 criteria for the classification of polyarteritis nodosa, Arthritis Rheum 33 (1990), 1088-1093. 
[2] J.C. Jennette, R.J. Falk, K. Andrassy et al., Nomenclature of systemic vasculitides: proposal of an international consensus conference, Arthritis Rheum 37 (1994), 187-192.

[3] B. Hellmich, O. Flossmann, W.L. Gross et al., EULAR recommendations for conducting clinical studies and/or clinical trials in systemic vasculitis: focus on anti-neutrophil cytoplasm antibody-associated vasculitis, Ann Rheum Dis 66 (2007), 605-617.

[4] R. Nowack, H. Lehmann, L.F. Flores-Suárez, A. Nanhou and F.J. Van Der Woude, Familial occurrence of systemic vasculitis and rapidly progressive glomerulonephritis, Am J Kidney Dis 34 (1999), 364-373.

[5] P. Fietta, Systemic vasculitides: immunogenetics and familial clustering, Clin Exp Rheumatol 22 (2004), 238-251.

[6] S. Borgmann and M. Haubitz, Genetic impact of pathogenesis and prognosis of ANCA - associated vasculitides, Clin Exp Rheumatol 22(suppl 36) (2004), S79-86.

[7] R. Harris and H.P. Jones, Polyarteritis nodosa in identical twins, Ann Phys Med 10 (1970), 241-242.

[8] R. Leff, W.V. Harrer, J.C. Baylis, L. Jackson and K. Faber, Polyarteritis nodosa in two siblings, Am J Dis Child 121 (1971), 67-70.

[9] J.D. Reveille, R.E. Goodman, B.O. Barger, R.T. Acton, Familial polyarteritis nodosa: a serologic and immunogenetic analysis, J Rheumatol 16 (1989), 181-185.

[10] M. Rottem, M.C. Frances, A.S. Fauci and G.S. Hoffmam, Familial Vasculitis: report of 2 families, J Rheumatol 21 (1994), $561-563$.

[11] J.C. Mason, M.R. Cowie, K.A. Davies, J.B. Schofield, J. Cambridge, M.J. Walport, Familial polyarteritis nodosa, Arthritis Rheum 37 (1994), 1249-1253.

[12] G. Barbiano di Belgiojoso, A. Genderini, R.A. Sinico et al., Acute renal failure due to microscopic polyarteritis with the same histological and clinical patterns in a father and his son, Contrib Nephrol 94 (1991), 107-114.

[13] L. Heuze-Claudot, B. Leroy, A. Chevailler et al., A familial ANCA-associated pulmonary-renal syndrome, Clin Exp Immunol 93(suppl 2) (1993), S40.

[14] C.F. Franssen, J.C. Ter Maaten and S.J. Hoorntje, Brother and sister with myeloperoxidase associated autoimune disease, Ann Rheum Dis 53 (1994), 213.

[15] R.A. Luqmani, P.A. Bacon and R.J. Moots, Birmingham Vasculitis Activity Score (BVAS) in systemic necrotizing vasculitis, Q J Med 87 (1994), 671-68.

[16] L. Guillevin, F. Lhote, M. Gayraud et al., Prognostic factors in polyarteritis nodosa and Churg-Strauss syndrome: a prospective study in 342 patients, Medicine 75 (1996), 17-28.

[17] L. Guillevin, B. Durand-Gasselin, R. Cevallos et al., Microscopic polyangiitis. Clinical and laboratory findings in eightyfive patients, Arthritis Rheum 42 (1999), 421-430.

[18] K.A. Boki, U. Dafni, G.A. Karpouzas, C. Papasteriades, A.A. Drosos and H.M. Moutsopoulos, Necrotizing vasculitis in Greece: clinical, immunological and immunogenetic aspects. A study of 66 patients, Br J Rheumatol 36 (1997), 1059-1066.

[19] K.B. Elkon, D.C. Sutherland, A.J. Rees, G.R.V. Hughes and J.R. Batchelor, HLA antigen frequencies in systemic vasculitis: increase in HLA-DR2 in Wegener's granulomatosis, Arthritis Rheum 26 (1983), 102-105.

[20] S.J.W. Spencer, A. Burns, G. Gaskin, C.D. Pusey and A.J. Rees, HLA class II specificities in vasculitis with antibodies to neutrophil cytoplasmic antigens, Kidney Int 41 (1992), 10591063.

[21] C. Papasteriades, D. Hatziyannakos, M. Siakotos et al., HLA antigens in microscopic polyarteritis with renal involvement, Dis Markers 13 (1997), 117-122.

[22] E.C. Hagen, C.A. Stegeman, J. D'amaro et al., Decreased frequency of HLA-DR13DR6 in Wegener's granulomatosis, Kidney Int 48 (1995), 801-805.

[23] M. Gencik, S. Borgmann, R. Zahn et al., Immunogenetic risk factors for anti-neutrophil cytoplasmic antibody (ANCA)associated systemic vasculitis, Clin Exp Immunol 117 (1999), $412-417$.

[24] N. Tsuchiya, S. Kobayashi, A. Kawasaki et al., Genetic background of japanese patients with antineutrophil cytoplasmic antibody-associated vasculitis: association of HLADRB $1 * 0901$ with microscopic polyangiitis, J Rheumatol 30 (2003), 1534-1540. 


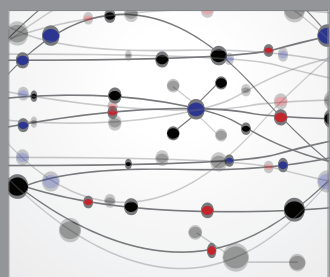

The Scientific World Journal
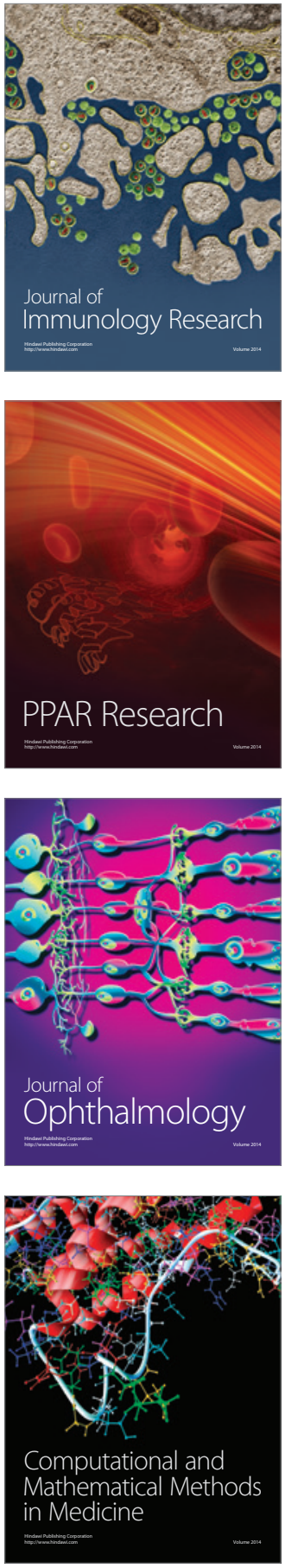

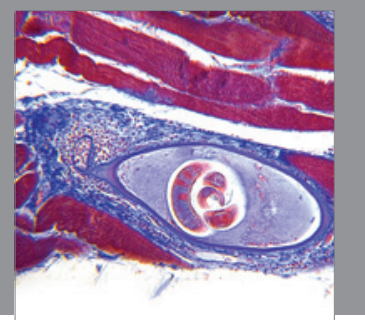

Gastroenterology

Research and Practice
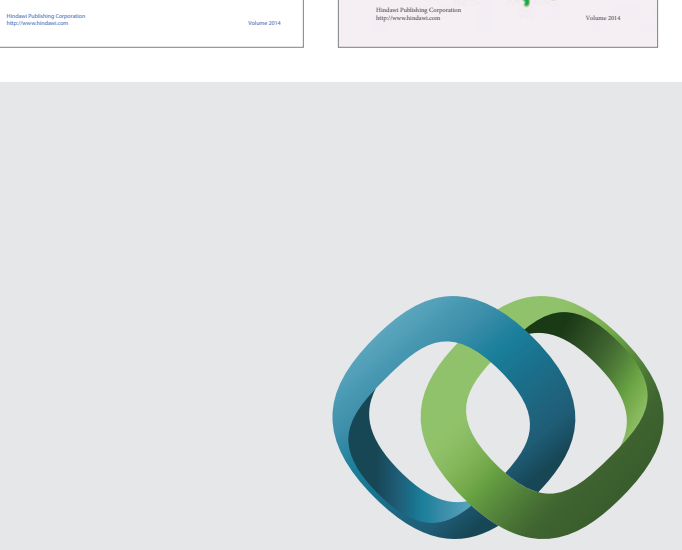

\section{Hindawi}

Submit your manuscripts at

http://www.hindawi.com
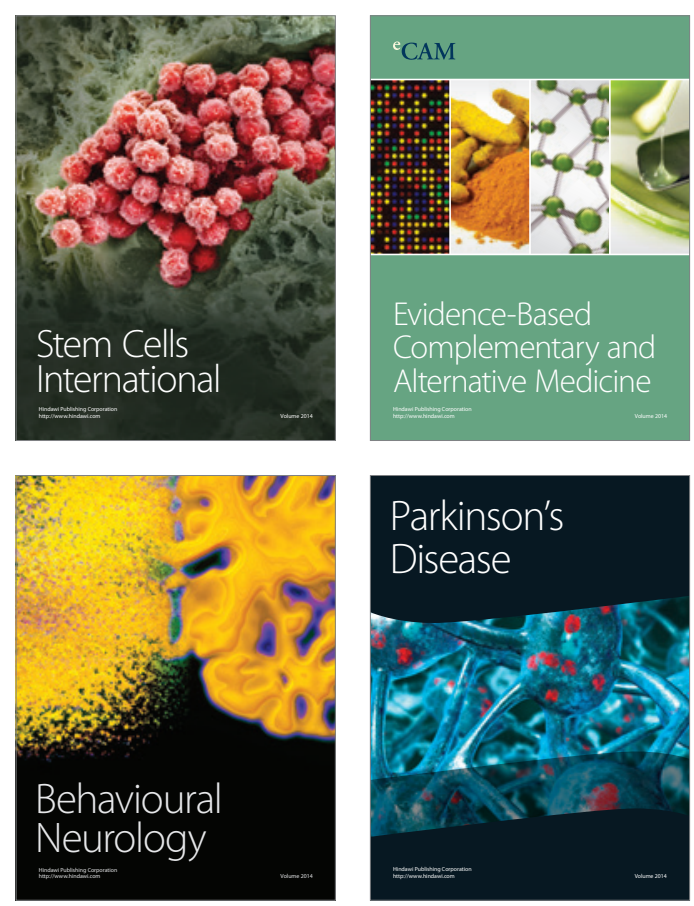

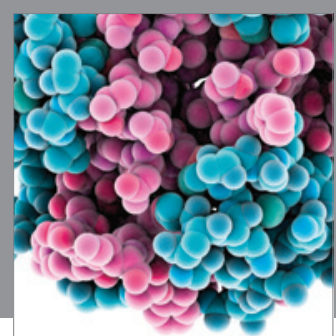

Journal of
Diabetes Research

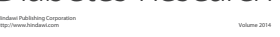

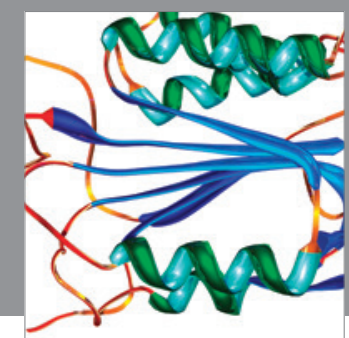

Disease Markers
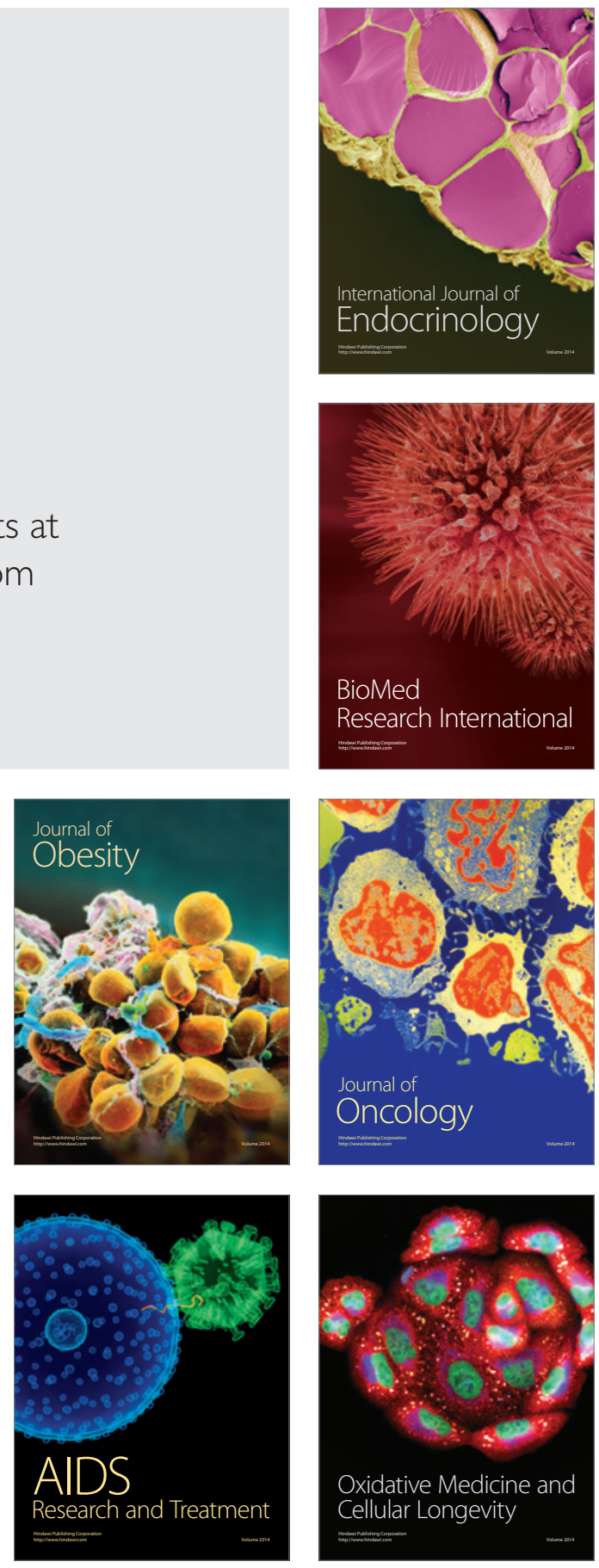